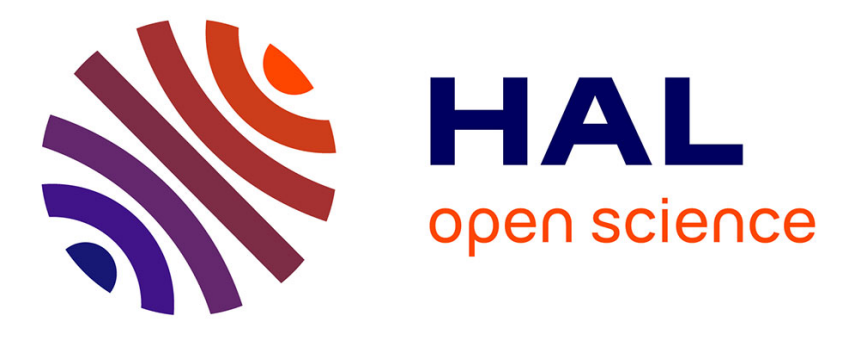

\title{
X-ray multilayer monochromator with enhanced performance
}

Jean-Michel André, Rabah Benbalagh, Robert Barchewitz, Marie-Françoise Ravet, Alain Raynal, Franck Delmotte, Françoise Bridou, Gwénäelle Julié, A. Bosseboeuf, René Laval, et al.

\section{To cite this version:}

Jean-Michel André, Rabah Benbalagh, Robert Barchewitz, Marie-Françoise Ravet, Alain Raynal, et al. X-ray multilayer monochromator with enhanced performance. Applied optics, 2002, 41 (1), pp.239-244. 10.1364/AO.41.000239 . hal-00874234

\section{HAL Id: hal-00874234 \\ https://hal-iogs.archives-ouvertes.fr/hal-00874234}

Submitted on 17 Oct 2013

HAL is a multi-disciplinary open access archive for the deposit and dissemination of scientific research documents, whether they are published or not. The documents may come from teaching and research institutions in France or abroad, or from public or private research centers.
L'archive ouverte pluridisciplinaire HAL, est destinée au dépôt et à la diffusion de documents scientifiques de niveau recherche, publiés ou non, émanant des établissements d'enseignement et de recherche français ou étrangers, des laboratoires publics ou privés. 


\title{
X-ray multilayer monochromator with enhanced performance
}

\author{
Jean-Michel André, Rabah Benbalagh, Robert Barchewitz, Marie-Françoise Ravet, \\ Alain Raynal, Frank Delmotte, Françoise Bridou, Gwénäelle Julié, Alain Bosseboeuf, \\ René Laval, Gérard Soullié, Christian Rémond, and Michel Fialin
}

\begin{abstract}
An x-ray multilayer monochromator with improved resolution and a low specular background is presented. The monochromator consists of a lamellar multilayer amplitude grating with appropriate parameters used at the zeroth diffraction order. The device is fabricated by means of combining deposition of thin films on a nanometer scale, UV lithography, and reactive ion etching. The performance of this new monochromator at photon energies near $1500 \mathrm{eV}$ is shown. (C) 2002 Optical Society of America

OCIS codes: $340.0340,300.6560$.
\end{abstract}

\section{Introduction}

During the past 20 years, multilayer interference mirrors (MIMs) have been implemented as monochromators in x-ray spectrometry for detection of lowatomic-number elements. ${ }^{1-5}$ They have also been used as monochromators in soft-x-ray reflectome-

J. M. André (andrejm@ccr.jussieu.fr), R. Benbalagh, and R. Barchewitz are with the Laboratoire de Chimie Physique, Matière et Rayonnement, Université Pierre et Marie Curie, Centre National de la Recherche Scientifique, Unité Mixte de Recherche 7416, 11 rue Pierre et Marie Curie, 75231 Paris Cedex 05, France. M.-F. Ravet, A. Raynal, F. Delmotte, and F. Bridou are with the Groupe de Physique des Films Minces, Laboratoire Charles Fabry de l'Institut d'Optique, Centre National de la Recherche Scientifique, Unité Mixte de Recherche 8501, Centre Scientifique Bâtiment 503, BP 147, 91403 Orsay, France. G. Julié, A. Bosseboeuf, and R. Laval are with the Institut d'Electronique Fondamentale, le Centre d'Études et d'Enseignement des Techniques Avancées de la Microélectronique, Centre National de la Recherche Scientifique, Unité Mixte de Recherche 8622, Université Paris Sud, Bâtiment 220, 91405 Orsay, France. G. Soullié and C. Rémond are with CEA-DAM/DRIF/DCRE/SDE, BP 12, 91680, Bruyères-le-Châtel, France. M. Fialin is with the Centre de Microanalyse CAMPARIS, Université Pierre et Marie Curie, Centre National de la Recherche Scientifique, Enseignement Superiéur Associée 7058, 75005 Paris, France. J.-M. André, G. Soullié, and C. Rémond are also with the Laboratoire pour l'Utilisation du Rayonnement Electromagnétique, Université Sud, Bâtiment 209 D, 91405 Orsay, France.

Received 16 November 2000; revised manuscript received 29 June 2001.

0003-6935/02/010239-06\$15.00/0

(C) 2002 Optical Society of America ters. ${ }^{6,7}$ Compared with Langmuir-Blodgett soapfilm monochromators, MIMs present numerous advantages:

(i) They offer high reflectivity and good stability under thermal and radiation loading and under vacuum.

(ii) They let one choose the nature of the constituents and thus optimize the peak reflectivity and avoid the regions of anomalous reflection. ${ }^{8}$

(iii) They allow the rejection of Bragg reflection orders by a judicious choice of the division parameter, ${ }^{9}$ which can prevent overlappings.

However, classic MIM monochromators have two main drawbacks:

(i) Their poor resolution, the resolving power being of the order of 20 near $100 \mathrm{eV}$.

(ii) The existence of a notable and nonlinear background that is due to the zeroth-order specular reflection.

These two drawbacks constitute an important impediment to quantitative analysis by soft-x-ray spectrometry, especially for trace elements.

In 1993 the principle of a multilayer x-ray monochromator with an improved resolution was given. ${ }^{10}$ As explained in further detail in this paper, this monochromator is based on the use of a lamellar multilayer amplitude grating (LMAG). The possibility of reducing simultaneously the specular background through the use of such a LMAG has also 
been recognized. ${ }^{11}$ The first evidence of the validity of these concepts has been obtained experimentally. ${ }^{12}$ Nevertheless, the parameters of the LMAGs used in the references cited above did not yet allow for considerable improvement in terms of reduction of bandpass and specular background.

Let us recall that the combination of a diffraction grating with a multilayer structure was initiated by Keski-Kuha ${ }^{13}$ and Jark ${ }^{14}$ to enhance the diffraction efficiency of gratings in the x-ray domain. The gratings were coated with multilayers, and improvement in reflectivity was actually observed. This method was then implemented by several teams to enhance the efficiency of $\mathrm{x}$-UV gratings working at nearnormal incidence. ${ }^{15-17}$ Etched multilayer mirrors with a lamellar profile were parallely developed by several groups, using different technologies for various applications: x-ray laser output couplers, diffraction gratings, polychromators, and so on. ${ }^{18-21}$

In this paper we present the results obtained with a plane LMAG suited for photon energy near $1500 \mathrm{eV}$. An important reduction of the bandpass and of the specular background has been observed at this energy. The improvements in the emission spectra of the Al-K $\alpha$ during use of this LMAG as monochromator with respect to the corresponding classic MIM monochromator are shown.

The paper is organized as follows: Section 2 is devoted to the principles of a multilayer monochromator with an improved resolution and a low specular background, based on a LMAG. Sections 3 and 4 deal with the techniques of elaborating on this new monochromator. Section 5 gives results of measuring the reflectivity of this monochromator recorded at $1500 \mathrm{eV}$ by means of the synchrotron radiation supplied by the Super-ACO (ACO is Anneau de Collision d'Orsay) storage ring of the LURE (Laboratoire pour l'Utilisation du Rayonnement Electromagnétique, Orsay) and in comparison presents the emission spectra of Al-K $\alpha$ recorded with the classic MIM monochromator and the LMAG monochromator. Section 6 presents conclusions and future objectives.

\section{Principle of the Lamellar Multilayer Amplitude Grating Monochromator}

To understand the principle of the LMAG monochromator, it is necessary at first to analyze the origin of the bandwidth and of the specular background of a classic MIM:

(i) Bandwidth. The bandwidth of a MIM is determined by the number of bilayers $N_{\text {eff }}$ that effectively participates in the diffraction process. The larger $N_{\text {eff }}$, the narrower the bandwidth of the multilayer monochromator. In practice, provided that the actual number of bilayers in the structure is large enough, this effective number $N_{\text {eff }}$ is limited by the depopulation of the incident wave through the photoabsorption process and the reflections at the interfaces. The bandwidth of the MIM tends asymptotically toward a nonzero limit when the actual number of bilayers increases.

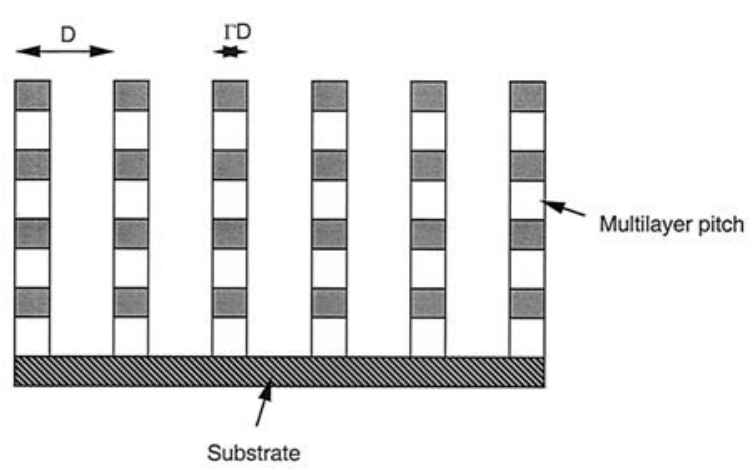

Fig. 1. Schematic of a LMAG monochromator.

(ii) Specular reflection background. In the x-ray domain the real part of the refractive index $n$ is generally less than unity and is usually written by means of a quantity called unit decrement $\delta$ as follows: $n=$ $1-\delta$. Consequently, a quasi-total-reflection phenomenon occurs for glancing angles $\theta$ smaller than the critical angle $\theta_{c} ; \theta_{c}$ is approximately equal to $\sqrt{2 \delta}$. For glancing angles larger than $\theta_{c}$, the reflectivity $R$ drops according to the following asymptotic behavior:

$$
R \rightarrow C\left[\left(\theta_{c}\right)^{4} / \sin ^{4} \theta\right],
$$

where $C$ depends on the photon energy and on the chemical composition of the medium. This equation shows that the specular reflectivity $R$ at a given energy and a given glancing angle larger than the critical angle will be reduced if $\theta_{c}$ is decreased. It is important to realize that both the photoattenuation coefficient and the unit decrement $\delta$ are proportional to the average density of the diffractive medium.

From the above considerations it appears that a method that makes it possible to decrease the average density of the medium leads both to a decrease of the bandwidth and to a reduction of the specular background. The reduction of the photoattenuation coefficient permits the increase of the number $N_{\text {eff }}$, and the reduction of the decrement $\delta$, that is, the reduction of the critical angle $\theta_{c}$, permits the reduction of the specular background.

We propose realizing the diminution of the average density of the diffractive medium by creating empty spaces in the multilayer structure. This can be done by means of etching the multilayer mirror according to the profile of a lamellar grating. We call the new device the LMAG. A schematic of such a device is given in Fig. 1 with the relevant parameters. It is important to understand that the LMAG is used under specular conditions, that is, as a Bragg mirror, and that the dispersion properties of an usual grating are not involved in the monochromator that we propose. The LMAG monochromator must be regarded as a lightened MIM, not as a dispersive grating.

\section{Fabrication of the Multilayer Mirror}

The results presented in this paper are obtained with a LMAG etched in a Mo-Si multilayer mirror consisting in 50 bilayers of molybdenum $3.4 \mathrm{~nm}$ thick 
(a)
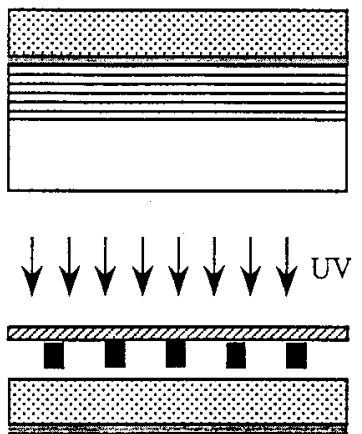

(b)

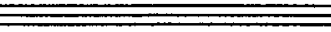

(c)

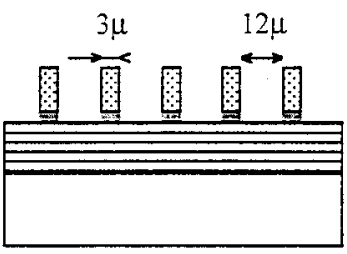

(d)

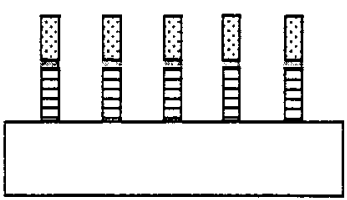

(e)

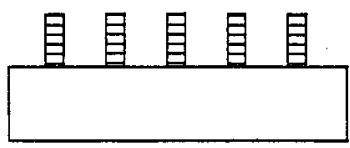

$\square \cdot$ Photoresist
圈 HMDS
曰 $\mathrm{Mo} / \mathrm{Si} \mathrm{ML}$
$\square \mathrm{Si}$
$\square \mathrm{glass}$
$\square \mathrm{Cr}$

Fig. 2. Process for fabricating the LMAG by UV lithography and RIE. Details of the different steps from (a)-(e) are given in the main text.

and of silicon $5.8 \mathrm{~nm}$ thick. The thickness of the layers is determined from $\mathrm{x}$-ray grazing reflectometry with the $\mathrm{K} \alpha$ emission line of copper (wavelength, $0.154 \mathrm{~nm}$ ). The mirrors are deposited by means of an ion-beam sputtering process. A $3-\mathrm{cm}$ Ion Tech, Inc., ion source fed with an $\mathrm{Ar}-\mathrm{H}_{2}(10 \%)$ gas mixture is mounted on a high-vacuum cryopumped chamber. The residual pressure in the deposition chamber is typically in the $10^{-9}$ mbar range, and the argon pressure during the deposition process is in the $10^{-4}$ mbar range. Sputtering of pure molybdenum $(99.95 \%)$ and silicon $(99.999 \%)$ is achieved with an ion beam of $30 \mathrm{~mA}$ and a voltage of $650 \mathrm{~V}$. The multilayer mirrors are deposited in a same batch onto six 1-in.-diameter $(1 \mathrm{in} .=2.54 \mathrm{~cm})$ flat and polished silicon substrates mounted on the rotating sample holder. Thus several identical samples can be used to adjust the parameters of the LMAG fabrication. One of the samples is kept as a reference for reflectometry measurements. Both targets are fitted on a double-faced holder that is alternatively turned under the ion beam in order to obtain an alternate de-

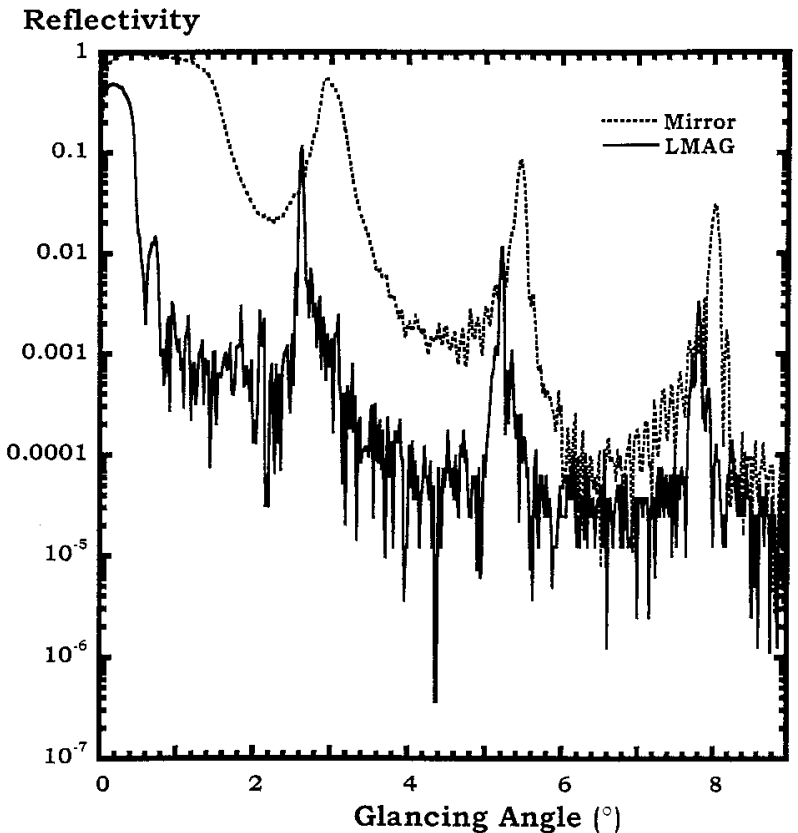

Fig. 3. Comparison between the experimental reflectivity and the glancing angle, of the LMAG monochromator and the corresponding MIM at the photon energy $1500 \mathrm{eV}$.

posit of both materials. The deposition rate and layer thickness are controlled in situ by a quartzcrystal balance whose oscillation is sensitive to the deposited mass for a given material. The controller is previously calibrated for each material by means of separately depositing molybdenum and silicon layers onto check samples and by measuring their thickness by x-ray grazing reflectometry as a function of the frequency increment given by the quartz balance.

\section{Fabrication of the Lamellar Multilayer Amplitude Grating}

A set of lamellar gratings with a grating pitch of $D=$ $15 \mu \mathrm{m}$ and a multilayer bar width $\Gamma D=3 \mu \mathrm{m}$ are fabricated in the Mo-Si MIMs by UV lithography and reactive ion etching (RIE). The total dimensions of the etched area are $20 \mathrm{~mm} \times 20 \mathrm{~mm}$. The process steps are schematically illustrated in Fig. 2. First, a thin layer of hexamethyldisilazane (HMDS), an adhesion promoter, is spread on the MIM surface. Then a $1.3-\mu \mathrm{m}$-thick positive photoresist (Shipley S1813) is spin-coated and baked at $95^{\circ} \mathrm{C}$ for $90 \mathrm{~s}$ on a hotplate [Fig. 2(a)]. After UV exposure with a standard contact aligner through a chromium-glass mask [Fig. 2(b)], the photoresist is developed in an aqueous alkaline solution (Shipley Microposit 351: $\left.\mathrm{H}_{2} \mathrm{O}, 1: 4\right)$ and hard baked at $120^{\circ} \mathrm{C}$ for $180 \mathrm{~s}$ [Fig. 2(c)]. Photoresist patterns are transfered in the MIM by RIE in a $\mathrm{SF}_{6}-\mathrm{CHF}_{3}-\mathrm{O}_{2}$ plasma [Fig. 2(d)]. Gas flow rate (10 SCCM SF 6 , 30 SCCM $\mathrm{CHF}_{3}, 10$ SCCM $\left.\mathrm{O}_{2}\right)$, rf power $(50 \mathrm{~W})$, and total pressure $(0.013$ mbar) are optimized to obtain both an anisotropic etching and a low etch rate $(25 \mathrm{~nm} / \mathrm{min})$ (SCCM denotes cubic centimeters per minute at STP). Etching rate is determined by in situ laser interferometry 


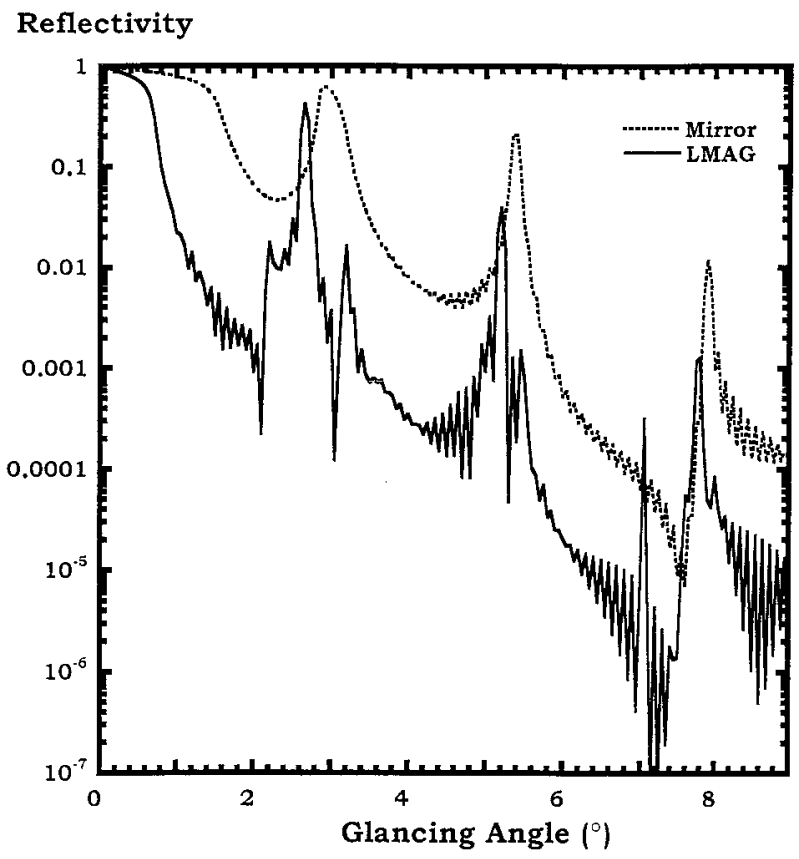

Fig. 4. Comparison between the theoretical reflectivity and the glancing angle, of the LMAG monochromator and the corresponding MIM at the photon energy $1500 \mathrm{eV}$. on test samples. Finally, the wafers are immersed in acetone to strip the resist, leading to a clean Mo-Si grating [Fig. 2(e)]. The patterning of the gratings by this process needs a chromium-glass lithographic mask with fine patterns on a large area. The grating on this mask is not strictly regular and must be treated as a patchwork of $5 \mathrm{~mm} \times 5 \mathrm{~mm}$ subgratings with slight misalignment errors. This imperfection results from the final stage of the mask fabrication process, which includes a step-and-repeat lithography.

\section{Characterization of the Lamellar Multilayer Amplitude Grating Monochromator}

Two types of characterization have been carried out. The first one performed with the synchrotron radiation of the Super-ACO storage ring (Orsay, France) has permitted the measurement of the absolute efficiency of the LMAG monochromator in comparison with that of the corresponding MIM at $1500 \mathrm{eV}$. The second one was conducted with a classic soft-x-ray tube equipped with an aluminum target emitting the $\mathrm{K} \alpha$ line; it illustrates the capability of the LMAG monochromator for x-ray spectrometry.

The experiment with synchrotron radiation was performed with the so-called MOGOTOX reflectometer setup on the SB3 beamline of the Super-ACO. The white radiation is monochromatized by means of

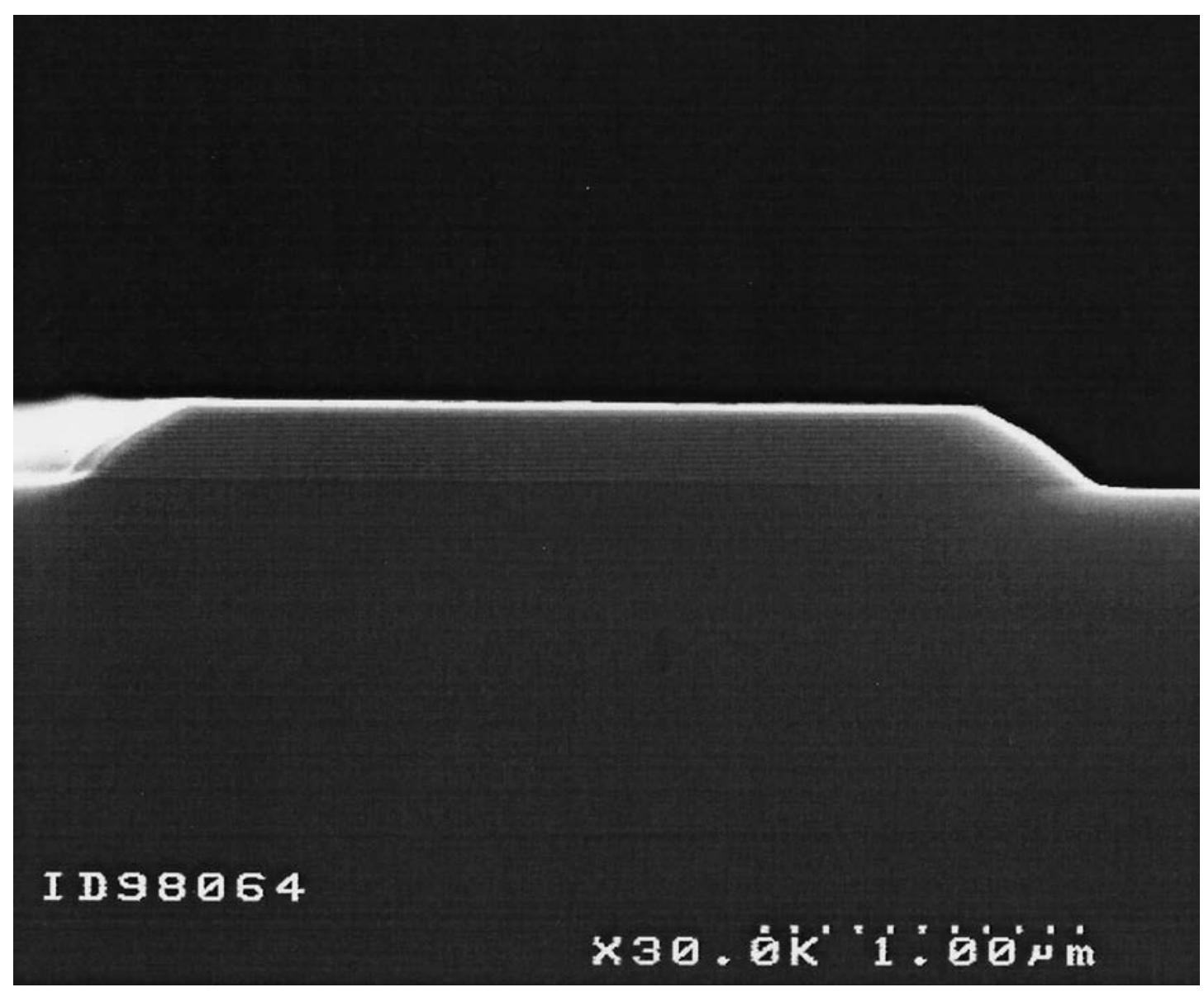

Fig. 5. SEM micrograph of our LMAG. 


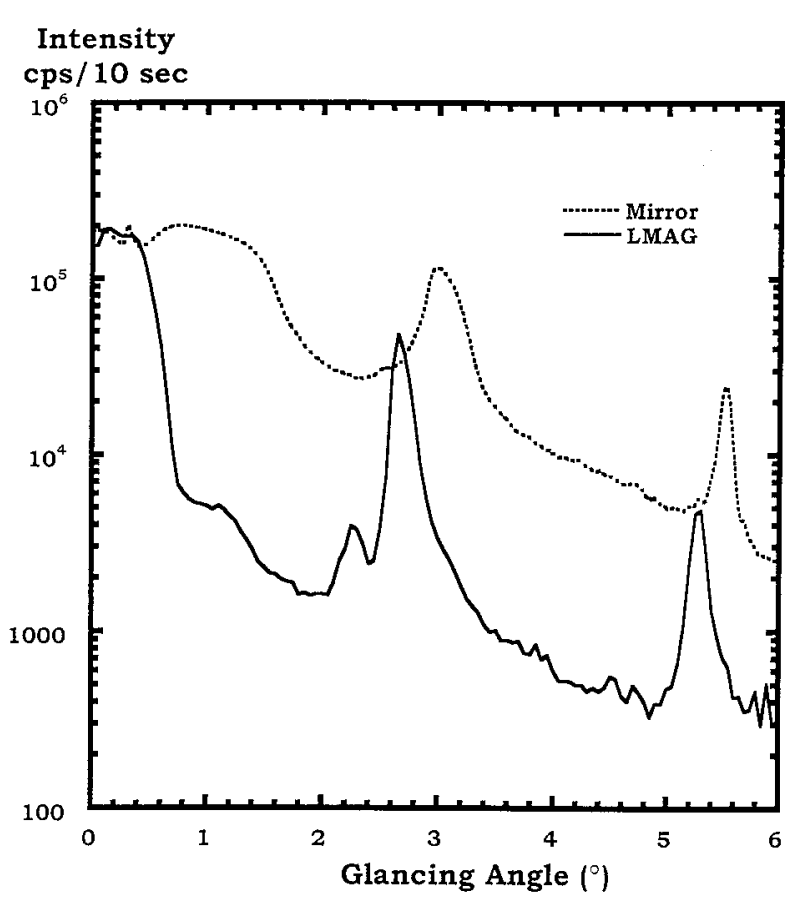

Fig. 6. Spectrum of an aluminum target recorded with the LMAG monochromator and the corresponding MIM. The Al-K $\alpha$ line is observed at the two first orders of Bragg diffraction.

a double-crystal monochromator equipped with beryl (1340) sheets, which gives a resolution of $0.5 \mathrm{eV}$ at $1500 \mathrm{eV}$. The LMAG monochromator is oriented with the grooves perpendicular to the incident radiation, that is, parallel to the light polarization. Figure 3 shows the absolute efficiency of the LMAG monochromator versus the glancing angle at $1500 \mathrm{eV}$ in comparison with the reflectivity of the corresponding MIM. One observes a large reduction of the bandpass and a considerable decrease of the specular background with passage from the MIM to the LMAG monochromator. The reduction of the bandwidth at the first-order Bragg peak is close to 5 .

One also notes a shift between the peaks recorded with the mirror and the LMAG monochromator. This can be understood as follows: The position of the Bragg peak depends on the average value of the optical index of the diffractive medium as a consequence of the correction of refraction [see Eqs. (26) of ref. 23]. Since this value is not the same for the mirror and for the LMAG, because this latter structure contains grooves whose optical index is equal to unity, there is a difference in the position of the peaks.

These measurements can be compared with theoretical forecasts obtained with the so-called modal method of calculation of grating efficiency ${ }^{24}$ (see Fig. 4). One notes that the theoretical peak efficiency of the LMAG monochromator is much larger than the measured one. This discrepancy can be attributed to several technical reasons:

- A thin layer of resist has likely not been removed from the top of the LMAG monochromator.
- The profile of the multilayer bars are not really rectangular but rather trapezoidal so that multilayer structure is lacking at the top of the multilayer bars. This is clear from the scanning electron microscope (SEM) micrograph (see Fig. 5).

Figure 6 shows the spectrum of the Al-K $\alpha$ recorded with the MIM and the LMAG mirror with a laboratory spectrometer. ${ }^{6}$ On notes that the specular background is considerably reduced with the LMAG monochromator with respect to the MIM and that the lines associated with the $\mathrm{Al}-\mathrm{K} \alpha$ emission are better resolved when the LMAG monochromator is used.

\section{Conclusion}

We have shown the possibility of reducing both the bandpass and the specular background of a multilayer x-ray monochromator, by using a LMAG in the specular condition. This has been achieved by means of combining the techniques of thin-film deposition with technologies borrowed from microelectronics [UV lithography, reactive ion etching (RIE)]. Calculations show that for the ultrasoft part of the spectrum, small $D$ values of the period of the LMAG (of the order of $1 \mu \mathrm{m}$ ) are required. Consequently, it would likely be necessary to implement direct writing by electron beam (electron lithography) to achieve this goal.

Our objectives now are to improve our fabrication methods in order to increase the efficiency of the LMAG monochromator and to develop such monochromators for the ultrasoft part of the spectrum. The improvement of the spectrometry for light chemical elements $(\mathrm{O}, \mathrm{N}, \mathrm{C}, \mathrm{B})$ with this kind of monochromator is our main goal in the future.

\section{References}

1. J. A. Nicolosi, J. P. Groven, D. Merlo, and R. Jenkins, "Layered synthetic microstructures for long wavelength x-ray spectrometry," Opt. Eng. 25, 964-970 (1991).

2. J. J. McGee, J. F. Slack, and C. R. Herrington, "Boron analysis by electron microprobe using $\mathrm{MoB}_{4} \mathrm{C}$ layered synthetic crystals," Am. Mineral. 76, 681-684 (1991).

3. D. S. Urch, "Soft x-ray spectroscopy," J. Phys. III France 4, 1613-1623 (1994).

4. B. Habulihaz, E. Martins, S. Gampblin, and D. S. Urch, "Chemical effects in soft x-ray spectra-even with multilayers: silicon L spectra using a 300 angströms device," X-Ray Spectrom. 25, 15-20 (1996).

5. C. Hombourger, P. Jonnard, J.-M. André, and J.-P. Chauvineau, "Use of layered synthetic microstructures for the quantitative analysis of light elements," X-Ray Spectrom. 28, 163 (1999).

6. M. Arbaoui, J.-M. André, P. Couillaux, and R. Barchewitz, "Versatile x-uv spectrogoniometer with multilayer interference mirrors," Rev. Sci. Instrum. 56, 2055-2058 (1985).

7. M. Arbaoui, R. Barchewitz, J.-M. André, Y. Lepêtre, and R. Rivoira, "Performances of a layered synthetic microstructure spectrogoniometer for characterictic x-ray lines," Opt. Eng. 25, 1207-1211 (1986).

8. R. Marmoret and J.-M. André, "Bragg reflectivity of layered synthetic microstructures in the x-ray anomalous scattering regions," Appl. Opt. 22, 17-19 (1983).

9. B. Pardo, T. Mégadémini, and J.-M. André, "X-UV synthetic 
interference mirror: a theoretical approach," Rev. Phys. Appl. 23, 1579-1597 (1988).

10. A. E. Sammar, J.-M. André, M. Ouahabi, B. Pardo, and R. Barchewitz, "Monochromateur multicouche à bande passante étroite pour le rayonnement X," C. R. Acad. Sci. Paris 316-II, 1055-1060 (1993).

11. M. Fialin, H. Rémy, J.-M. André, J.-P. Chauvineau, F. Rousseaux, M.-F. Ravet, D. Decanini, and E. Cambril, "Extending the possibilities of soft x-ray spectrometry through the etching of layered synthtic microstructure monochromators," X-Ray Spectrom. 25, 60-65 (1996).

12. A. Sammar, K. Krastev, J.-M. André, R. Barchewitz, and R. Rivoira, "Narrow bandpass multilayer x-ray monochromator," Rev. Sci. Instrum. 68, 2969-2972 (1997).

13. R. A. M. Keski-Kuha, "Layered synthetic microstructure technology considerations for the extreme ultraviolet," Appl. Opt. 23, 3534-3537 (1984).

14. W. Jark, "Enhancement of diffraction grating efficiencies in the soft x-ray region by a multilayer coating," Opt. Commun. 60, 201-205 (1986).

15. J. C. Rife, W. R. Hunter, T. W. Barbee, and R. G. Cruddace, "Multilayer-coated blazed grating performance in the soft x-ray region," Appl. Opt. 28, 2984-2986 (1989).

16. R. G. Cruddace, T. W. Barbee, J. C. Rife, and W. R. Hunter, "Performance of a tungsten/carbon multilayer-coated, blazed grating from 80 to $1700 \mathrm{eV}$," Phys. Scr. 41, 396-399 (1990).

17. A. M. Hawryluk, N. M. Ceglio, D. G. Stearns, K. Danzmann, M. Kuhne, P. Müller, and B. Wende, "Soft x-ray beamsplitters and highly dispersive multilayer mirrors for use as soft x-ray laser cavity components," in Multilayer Structures \& Laboratory
X-Ray Laser Research, N. M. Ceglio and P. Dhez, eds., Proc. SPIE 688, 81-90 (1986).

18. H. Berrouane, J.-M. André, R. Barchewitz, C. Khan Malek, and R. Rivoira, "Soft x-ray multilayer gratings with subhalfmicron period," Opt. Commun. 76, 111-115 (1990).

19. V. A. Chernov, N. I. Chkhlao, N. V. Kovalenko, and S. V. Mytnichenko, "Fabrication and performance characteristics of a Ni/C multilayer grating for hard x-rays," Nucl. Instrum. Methods Phys. Res. A 359, 138-140 (1995).

20. K. Krastev, F. Le Guern, K. Coat, R. Barchewitz, J.-M. André, M.-F. Ravet, E. Cambril, F. Rousseaux, and P. Davi, "Spectral sampling of a soft x-ray tube by a lamellar multilayer grating in standard and conical mountings," Nucl. Instrum. Meth. Phys. Res. A 368, 533-542 (1996).

21. M. Jergel, P. Mikulik, E. Majkova, S. Luby, R. Senderak, E. Pincik, M. Brunel, P. Hudek, I. Kostic, and A. Konecnikova, "Structural characterisation of lamellar multilayer gratings by x-ray reflectivity and scanning electron microscopy," J. Phys. D 32, A220-A223 (1999).

22. J.-M. André, A. Sammar, R. Barchewitz, C. Bizeuil, P. Stemmler, and B. Villette, "X-UV lamellar multilayer amplitude gratings,” J. X-ray Sci. Technol. 8, 171-193 (1998).

23. A. Sammar, J.-M. André, and B. Pardo, "Diffraction and scattering by lamellar amplitude multilayer gratings in the X-UV region," Opt. Commun. 86, 245-254 (1991).

24. K. Krastev, J.-M. André, and R. Barchewitz, "Further applications of a recursive modal method for calculating the efficiencies of X-UV multilayer gratings," J. Opt. Soc. Am. A 13, 2027-2033 (1996) 